\title{
Precision Freehand Sculpting of Bone
}

\author{
Gabriel Brisson ${ }^{1}$, Takeo Kanade ${ }^{1}$, Anthony DiGioia ${ }^{2}$, Branislav Jaramaz ${ }^{2}$ \\ ${ }^{1}$ The Robotic Institute, Carnegie Mellon University, Pittsburgh PA, USA \\ \{brisson, tk\}@cs. cmu . edu \\ ${ }^{2}$ The Institute for Computer Assisted Orthopaedic Surgery, The Western Pennsylvaia \\ Hospital, Pittsburgh, PA, USA \\ \{tony, branko\} aicaos.org
}

\begin{abstract}
The Precision Freehand Sculptor (PFS) is a compact, handheld, intelligent tool to assist the surgeon in accurately cutting bone. A retractable rotary blade on the PFS allows a computer to control what bone is removed. Accuracy is ensured even though the surgeon uses the tool freehand. The computer extends or retracts the blade based on data from an optical tracking camera. Three users used each of three PFS prototype concepts to cut a faceted shape in wax. The results of this experiment were analyzed to identify the largest sources of error.
\end{abstract}

\section{Introduction}

Artificial joint replacements must be installed accurately to ensure proper joint biomechanics. Proper installation requires cutting precise shapes out of bone. The Precision Freehand Sculptor (PFS) is a compact, handheld, intelligent tool to assist the surgeon in accurately cutting these shapes (Figure 1). A retractable rotary blade on the PFS allows a computer to control what part of the bone is removed, ensuring accurate results even though the surgeon uses the tool freehand. The surgeon simply glides the tool over the bone surface, where the tool cuts away only the bone that should be removed.

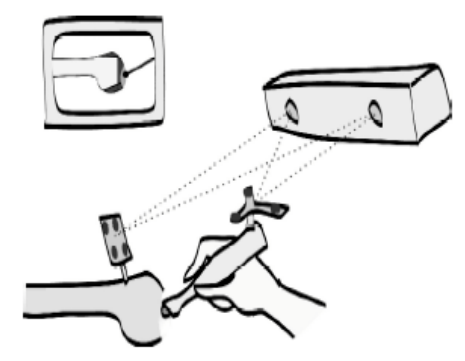

Fig. 1. The PFS system consists of a handheld tool, a tracking system and a display.

To control the tool's retractable blade, the computer compares the tool's current position to a programmed "target shape" for the cut bone. The positions of the tool and the bone are reported to the computer by an optical tracking camera. 
A computer display shows the tool and current bone surface, as well as the target shape for the bone. It also shows how close the cutter is to the target shape. This allows surgeons to monitor the progress of the cutting task.

Two prototype PFS versions have been completed. To evaluate their performance, each was used by three users to cut a faceted shape in wax. In addition, each user used the more advanced prototype without blade retraction, guided only by the display screen. The resultant surface was digitized with a touch-probe scanner to evaluate the accuracy of the tools. Secondary information such as maximum tool velocity and acceleration was also gathered, and users were observed and surveyed on their use of the display.

The results from this experiment were analyzed to identify major sources of error. This paper focuses on modeling error, which is error in the computer's internal model of where the bone has been cut. Software speed was found to be the largest source of modeling error, while calibration and tracking accuracy also contributed.

We expect the PFS to enable accurate surgery through smaller incisions. A PFS mechanism smaller than the current designs would be ideal for working in small, cramped incisions because the cutter is closely guarded, and because it ensures accuracy even when the surgeon does not have a clear view.

In contrast to robots such as RoboDoc [1] and Acrobot [2], the PFS has a smaller footprint and does not require the bone to be fixed in place. It also features the inherent safety of not being able to move on its own, other than the small motion of blade extension.

The PFS can be seen as an extension of surgical navigation [3]. The PFS extends the capabilities of navigation to allow preparation of more complex shapes. Like navigation, the PFS has the potential to reduce incision size by replacing direct visualization with on-screen visualization [4].

\section{System Description}

The PFS system consists of a handheld tool, an optical tracking system, a control computer, and a display monitor (Figure 1). Two PFS handheld tool prototypes have been developed.

The earlier "clutch tool" prototype (Figure 2) has a fully exposed spherical blade. The blade starts and stops rotation to control cutting. The clutch tool features a clutch which engages and disengages the blade from the drive shaft. Unfortunately it does not include a brake to stop blade rotation. As a result the blade spins up very fast but takes several seconds to spin down.

The "shaver" prototype (Figure 2) is more advanced. It features a cylindrical rotating blade which extends and retracts behind a guard. The axis of the blade is perpendicular to the long axis of the tool. The blade extends $0.64 \mathrm{~mm}(0.025 \mathrm{in})$ beyond the guard to cut, and retracts $0.64 \mathrm{~mm}(0.025 \mathrm{in})$ behind the guard to stop cutting, so the transition can be fast and not interfere with the surgeon's motion. Since the blade retracts such a small distance, the opening in the guard must be small to prevent it from cutting. The guard opening is $7.82 \mathrm{~mm}(0.308 \mathrm{in})$, which will 
prevent a sphere with a radius larger than $12.37 \mathrm{~mm}(0.487 \mathrm{in})$ from contacting the retracted blade. The blade diameter is $15.2 \mathrm{~mm}(0.6 \mathrm{in})$.
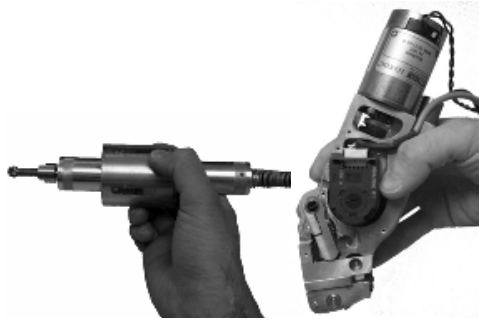

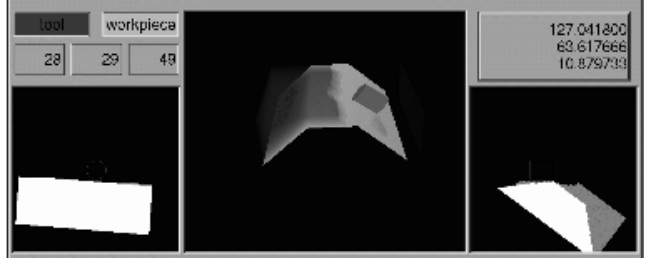

Fig. 3. PFS computer display

Fig. 2. Clutch tool (left) and shaver tool (right)

Both tool prototypes use the same software and tracking system. The position of the tool and bone is tracked by an Optotrak ${ }^{\mathrm{TM}}$ camera system (Northern Digital Inc, Waterloo Canada). The nominal accuracy of the Optotrak is $0.1 \mathrm{~mm}$ RMS at each LED, but small rotational errors can create larger errors away from the marker, e.g. in calculating the position of the blade. With the configuration of markers used in this experiment, we expect the tracking error to be approx $+/-0.3 \mathrm{~mm}$, and the tracking latency to be about $4 \mathrm{~ms}$.

The shape that the system is instructed to cut, called the target shape, is determined by the implant shape and its planned position. Planning options include preoperative planning on CT scans [5], positioning based on anatomical landmarks the surgeon locates [6], or statistical atlas models of the bone [7]. The PFS is compatible with any of these approaches. It is a tool for executing whatever plan is given it.

The computer display (Figure 3) contains a 3D view, two cross-sectional views, and a bargraph (bottom) that displays the closest distance between the tool's cutter and the target shape. The boxes labeled "tool" and "workpiece" are green when the corresponding Optotrak marker is in view and red when it is not.

The computer maintins a model of the bone surface as it's being cut. In the 3D view, the current bone surface is shown transparently above the target shape. As its distance to the target surface is reduced, the current bone surface changes from red to orange $(2 \mathrm{~mm})$ to green $(0.6 \mathrm{~mm})$ and finally disappears in places where the target surface is penetrated. In the $3 \mathrm{D}$ view the bone stays fixed and the blade of the tool moves around it. The user may rotate the $3 \mathrm{D}$ view by stepping on a footpedal and using the optically tracked tool as an intuitive "3D mouse".

The cross-sectional views show the target shape in white and the waste bone in gray. The cross section planes pass through the center of the blade and are fixed in orientation with respect to the tool. This arrangement dictates that the outline of the tool remains stationary and the bone moves around it, in contrast to the $3 \mathrm{D}$ view.

\section{Experimental Setup}

Three users used each of the two tool prototypes, the "shaver" and "clutch tool", described above to cut a simple faceted target shape in wax. Additionally each user 
used the shaver tool without blade retraction, where cutting was guided only by the screen display in order to gauge necessity of the blade retraction for accurate cutting. The tool used in this mode is referred to as the "navigated shaver". The cut workpiece was digitized to determine accuracy. The user's motion through each cutting trial was recorded, from which user speeds and accelerations were extracted.

The workpiece used (Figure 4) was CNC validation wax (MSC Industrial Supply Co., Melville, NY, USA), pre-cut to limit bulk material removal without giving away the shape of the underlying target surface. A step milled in each end of the workpiece defined a reference plane. The workpiece was mounted with three screws to a steel holder (Figure 4) to which the tracking system marker was attached. Registration pins on the holder allowed the wax in each trial to be repeatably positioned with respect to the marker.
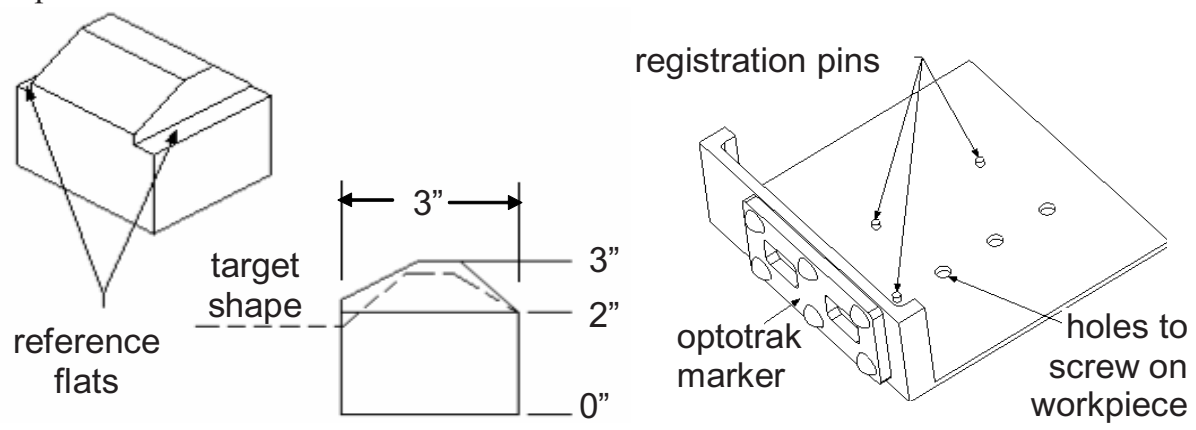

Fig. 4. Wax workpiece (left) and workpiece holder (right).

The cut workpieces were scanned on a Renishaw Cyclone Series 2 touch-probe scanner (nominal accuracy $0.002 \mathrm{in}=0.051 \mathrm{~mm}$ ) on a $0.932 \mathrm{~mm}(0.0367 \mathrm{in})$ pitch grid, resulting in approximately 1500 samples per wax block. Since the uniform grid produces more samples per surface area on the diagonal facets, points were weighted by the arc-cosine of the facet angle when calculating the accuracy of results.

Subjects were already familiar with the PFS concept. Each subject was given a short orientation based on a written script. Subjects were instructed to try to be as accurate as possible, and were responsible for deciding when to finish the trial. After each trial, subjects completed a survey about their usage of the tool and user interface. Each subject performed the trials in a different order. After initial trials were complete, an error was discovered which required navigated shaver trial to be redone.

While the subject used the tool, the software recorded the blade position, retraction status, and loop time at each software loop. The final 3D model of the cut workpiece surface was also recorded for comparison with the ground truth determined by the touchprobe scanner.

\section{Results}

The histograms in Table 1 summarize the accuracy results. The left column profiles the error between the actual cut surface and the target surface, with negative numbers 
indicating that the actual surface was cut too deep. The right column profiles the error between the computer 3D model of the cut surface and the actual cut surface, in effect how much the computer's model of what was cut differs from reality. Negative numbers indicate that the actual surface was cut deeper than the computer model. Note that the clutch tool results are on a larger scale than the others.

Error can be divided into execution error and modeling error. Execution error is that which the computer was aware of but could not prevent: it is reflected in the computer 3D model. Modeling error is that which the computer was unaware of: it is the difference between the final computer model and the actual cut surface, as recorded in the left column of Table 1. As it seems most efficient to worry about execution error only once accurate modeling is achieved, we will discuss execution error briefly and focus on modeling error.

Table 1. Accuracy results.

Lirror. Difference between scanned surface \& target surface(mrn)
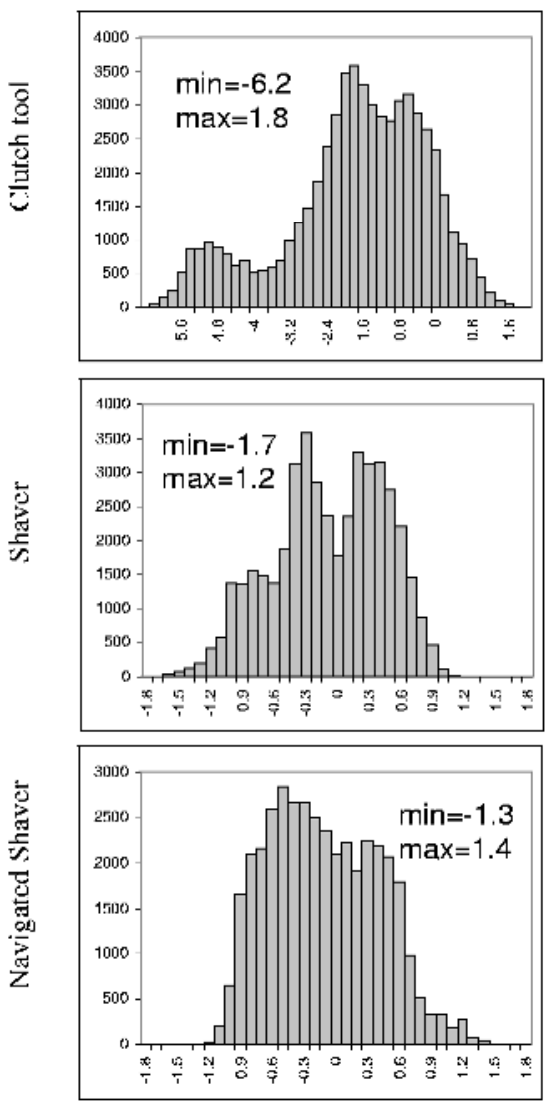

Modeling error. Difference between scanned surface \& computer model(mm)
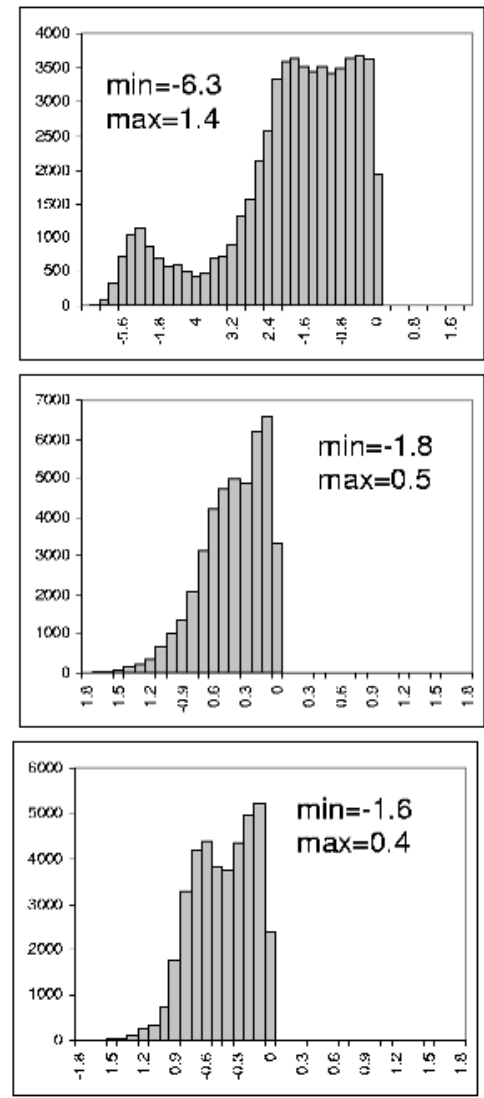


\subsection{Execution Error}

Execution error in this system has three main causes.

1. Blade retraction speed. (This does not apply to the navigated shaver.) Although the retraction speed of the current prototypes can certainly be improved, almost any PFS mechanism will have a non-negligible retraction time. The solution is for the software to look ahead and retract the blade before the tool reaches an area that shouldn't be cut. This is a focus of future work.

2. Lack of partial blade retraction. (This only applies to the shaver.) The software currently extends the blade fully or not at all. For example, if the blade is retracted and passes over an area that needs $0.5 \mathrm{~mm}$ removed from it, the software will not extend the blade because it extends $0.64 \mathrm{~mm}$ and would thus violate the target shape. This will be corrected in future software versions.

3. Resolution of target shape coloring. When the current surface of the workpiece is cut to within $0.6 \mathrm{~mm}$ of the target surface, the display in that area turns green. Where the surface is cut beyond the target shape, the display turns white. Obviously the subject cannot tell, for a point in that is colored green, exactly what the distance is. The bargraph provides higher resolution distance measurement but measures from whatever part of the blade is closest and doesn't indicate which part that is. Although this is most limiting for the navigated shaver, it is used even when the blade is operational to find areas which need to be cut. The display will be changed so that green represents a smaller range.

\subsection{Modeling Error}

There are four main sources of modeling error in this system. Below they are described and their relationship to the observed modeling error is discussed.

1. Blade retraction modeling. The delay in blade retraction is not currently modeled in software, and thus can contribute to modeling inaccuracy. When the software tells the blade to retract, it instantly switches to updating the cut bone model based on the retracted position of the blade, regardless of the blade's actual position. This does not affect the navigated shaver, since the blade never retracts. The similarity between the modeling error of shaver and navigated shaver indicates that this is not a significant source of modeling error.

2. Workpiece and/or tool calibration. The calibration matrix describes where the workpiece or tool is with respect to its tracking marker. Inaccuracy will result in the tool not cutting where the computer expects. The most likely source of calibration error is the optical-tracking-based procedure used to register the workpiece and tool initially. Since each new workpiece is registered with the positioning pins on the steel holder, and since the tool registration remains constant through trials, errors due to optical tracking calibration should persist through multiple trials. Thus bad calibration would produce results that are repeatable but not accurate. Figure 5 shows the combined histogram of modeling error for all shaver and navigated shaver trials broken down by facet of the target shape. The 
modeling error for each facet is more tightly clustered than the aggregate distribution of modeling error, indicating the influence of calibration error.

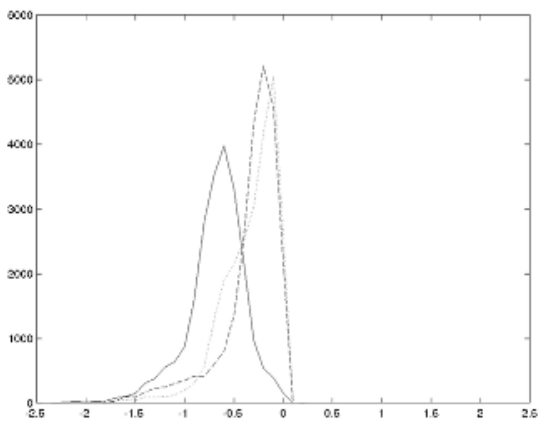

Fig. 5. Combinedshaver/navigated shaver modeling error $(\mathrm{mm})$ broken down by facet.

3. Software cycle time. Each software cycle the computer updates the model of the cut workpiece based on the current position of the tool. Any motion of the tool in between software updates is not reflected in the model of the current workpiece surface. Tables 2 and 3 summarize the software loop times and tool velocities recorded throughout trials. Even for a modest loop time of $80 \mathrm{~ms}$ and a modest speed of $0.1 \mathrm{~m} / \mathrm{s}$, the distance of the shaver blade between software iterations is $8 \mathrm{~mm}$, which for the $15.2 \mathrm{~mm}$ diameter blade traveling across the surface will result in a modeling error of $1.1 \mathrm{~mm}$. This problem is mitigated with the shaver mechanism because the shaver's guard limits the depth of cut so that an area must be passed over multiple times to cut a significant depth. The clutch tool, on the other hand, can make a straight plunging cut and come back up before the software registers its position. This accounts for the difference in modeling error between the shaver/navigated shaver and the clutch tool. Note that modeling error is almost exclusively negative: the computer underestimates how much material has been cut. This is consistent with the modeling errors that can result from slow software.

Table 2. Distribution of software loop times observed in trials.

\begin{tabular}{|l|l|l|l|}
\hline Software loop time: & $40-80 \mathrm{~ms}$ & $80-160 \mathrm{~ms}$ & $160-310 \mathrm{~ms}$ \\
\hline \# of software cycles: & $85912(73 \%)$ & $31866(27 \%)$ & 10 \\
\hline
\end{tabular}

Table 3. Distribution of tool velocities observed in trials.

\begin{tabular}{|l|l|l|l|}
\hline Tool velocity: & $0-0.1 \mathrm{~m} / \mathrm{s}$ & $0.1-0.5 \mathrm{~m} / \mathrm{s}$ & $0.5-0.9 \mathrm{~m} / \mathrm{s}$ \\
\hline \# software cycles, (nav)shaver: & $131367(85 \%)$ & $22339(15 \%)$ & 150 \\
\hline \#software cycles, clutch & $35510(88 \%)$ & $4869(12 \%)$ & 40 \\
\hline
\end{tabular}


4. Tracking system accuracy. We expect tracking accuracy in this experiment to be around $+/-0.3 \mathrm{~mm}$. The modeling error seen in the shaver and navigated shaver is small enough to be affected by tracking errors but large enough that tracking error is not the dominant effect. The negative bias of the modeling error supports this conclusion: for a calibrated tracking system, error is usually expected to be centered around zero.

\section{Conclusion}

The largest sources of modeling error in this experiment were calibration error followed by software speed for the shaver and navigated shaver trials. In the clutch tool trials software speed dominated.

Although the guarded mechanism of the shaver mitigates the problems of slow software, future guarded versions of the PFS will attempt to cut more aggressively than the current shaver and thus will be more affected by software speed. Better synchronization with the tracking system will be necessary to improve software speed and achieve accurate results. Future mechanical prototypes should be designed with calibration specifically in mind.

Improvements to the software to minimize execution error will also be necessary. Some of the execution error problems identified are easy fixes, whereas others, such as predictive retraction of the blade, are difficult and open-ended problems.

\section{References}

1. Taylor, et al: An Image-Directed Robotic System for Precise Orthopedic Surgery. IEEE Transactions on Robotics and Automation, 10(3), 1994, 261-274

2. Jakopec, et al: The First Clinical Application of a "Hands-On" Robotic Knee Surgery System. Computer Aided Surgery, 6, 2001, 329-339

3. Simon, D.A., et al: Development and Validation of a Navigational Guidance System for Acetabular Implant Placement. CVRMed-MRCAS'97, 583-592

4. Levinson, T.J., Moody, J.E., Jaramaz, B., Nikou, C., DiGioia, A.M.: Surgical Navigation for THR: A Report on Clinical Trial Utilizing HipNav. MICCAI2000, 1185-1187

5. Jaramaz, B., Nikou, C., Simon, D.A., DiGioia, A.M. III: Range Of Motion After Total Hip Arthroplasty: Experimental Verification Of The Analytical Simulator. CVRMedMRCAS'97, 573-582

6. Leitner, F., et al: Computer-Assisted Knee Surgical Total Replacement. CVRMedMRCAS'97, 629-637

7. Fleute, M., Lavallee, S. Julliard, R.: Incorporating a statistically based shape model into a system for computer-assisted anterior cruciate ligament surgery. Medical Image Analysis, 3(3), 1999, 209-222 